\title{
"EDUCACIÓN CON TEORÍA». REVISIÓN PEDAGÓGICA DE LAS RELACIONES ENTRE LA TEORÍA Y LA PRÁCTICA EDUCATIVA $^{1}$
}

\author{
"Education with theory". A pedagogical review \\ of the theory-practice relationships in education
}

\author{
"L'Éducation avec théorie". Révision des relations \\ entre la théorie et la pratique éducative
}

\begin{abstract}
Fernando GiL CANTERO ${ }^{1}$
Universidad Complutense de Madrid. Facultad de Educación, Centro de Formación del Profesorado. Departamento de Teoría e Historia de la Educación. c/ Rector Royo Villanova, s/n. Ciudad Universitaria. 28040 Madrid. Correo-e: gcantero@edu.ucm.es
\end{abstract}

Fecha de recepción: enero de 2011

Fecha de aceptación definitiva: abril de 2011

Biblid [(1130-3743) 23, 1-2011, 19-43]

1. Texto elaborado dentro del Proyecto de Investigación I+D, Referencia: EDU2010-17367, Secretaría de Estado de Investigación, Ministerio de Ciencia e Innovación, titulado: “Origen y desarrollo del conocimiento teórico de la educación en España". I.P.: Teresa Rabazas. Una primera versión se presentó, como conferencia, en el Seminari Pensament Pedagógic organizado por el Departament de Teoria i Història de l'Educació, Facultat de Pedagogia, Universitat de Barcelona. Me gustaría agradecer a los organizadores del Seminario (Ana Ayuste y Conrad Vilanou) y a los asistentes, quienes con sus preguntas me ayudaron a pensar mejor este tema. 


\section{RESUMEN}

Las relaciones entre la teoría y la práctica educativa abarcan tres áreas principales. Por un lado, se refieren a la enseñanza, a la transmisión de contenidos de aprendizaje. Por el otro, estas relaciones pertenecen al ámbito de los juicios educativos o las actividades de asesoramiento pedagógico. Por último, se relacionan con el ámbito de la investigación pedagógica y su relación con la práctica. El artículo plantea que es preciso diferenciar la teoría necesaria para la investigación pedagógica de la teoría necesaria para la práctica educativa. El texto también propone analizar críticamente las posiciones postfundacionalistas para reivindicar algunas de las aportaciones de la perspectiva moderna de la epistemología.

Palabras clave: teoría educativa, práctica educativa, investigación educativa, epistemología, postfundacionalismo.

\section{SUMMARY}

The theory-practice relationships in education cover three main areas. On the one hand, they refer to teaching, to the transmission of learning contents. On the other hand, those relationships pertain to the range of educational judgements or counselling activities. Finally, they relate with the field of educational research and its relationship to practice. The article argues that it is required to distinguish the theory needed for educational research, from the theory needed for educational practice. The text aims as well to analyze critically postfoundationalist's positions to vindicate some of the contributions of the modern perspective of epistemology.

Key words: educational theory, educational practice, educational research, epistemology, postfoundationalism.

\section{SOMMAIRE}

Les relations entre la théorie et la pratique éducative renferment trois domaines principaux. D'une part, elles se réfèrent à l'enseignement, à la transmission de contenus d'apprentissage. En outre, ces relations appartiennent au domaine des jugements éducatifs ou aux activités de conseil pédagogique. Finalement, elles ont de la relation avec le domaine de la recherche en éducation et sa relation avec la pratique. L'article fait valoir qu'il est nécessaire de distinguer la théorie nécessaire pour la recherche en éducation, de la théorie nécessaire pour la pratique éducative. Le texte vise également à analyser de façon critique les positions postfondationalistes pour réclamer quelques des contributions de la perspective moderne de l'épistémologie.

Mots clés: théorie de l'éducation, pratique éducative, recherche en éducation, épistémologie, postfondationalisme. 


\section{INTRODUCCIÓN. ESTADO DE LA CUESTIÓN}

El tema de la relación entre la teoría y la práctica constituye probablemente uno de los problemas centrales de la pedagogía y, en general, del pensamiento educativo en nuestro ámbito nacional e internacional ${ }^{2}$. Al establecer una agenda de tareas para el futuro que permita relacionar la teoría de la educación con las disciplinas pedagógicas afines (lo que nosotros denominaríamos ciencias de la educación), Uljens sitúa como tercer "problema resolver cómo debería ser la relación entre la teoría y la práctica educativa" (2001, 299). Wiliam reconoce, por su parte, que "(c)asi desde su aparición como un campo de investigación, la investigación educativa ha sido criticada por su falta de relevancia para la práctica y, en particular, por enfatizar la búsqueda de comprensiones teóricas a expensas de consideraciones prácticas” (Wiliam, 2008) ${ }^{3}$.

Aunque no es posible ocuparnos de todos los detalles de este tema, parece conveniente, sin embargo, establecer un cierto orden o clasificación inicial. Es habitual así diferenciar dos grandes ámbitos donde se manifiesta, de forma diferente, el problema de la relación entre la teoría y la práctica ${ }^{4}$.

En primer lugar, tenemos la práctica de los docentes como expresión operativa de concepciones pedagógicas. Aquí el problema de la práctica ha de entenderse como prueba de ajuste técnico y valorativo, de carácter docente, también educador, en situaciones de aula, o de educación no formal, buscando una enseñanza educativa. Referirse aquí a la relación entre teoría y práctica consiste, principalmente, en lo que, en un influyente texto, Ball y Forzani han denominado "la dinámica instructiva interna de la educación" $(2007,530)$. De lo que se trata es

2. Edmonds y Candy, 2010; González Pérez, 2010; Jover y Thoilliez, 2010; Winkle-Wagner, Hunter y Hinderliter, 2009; González Faraco y Gramigna, 2009; Wiliam, 2008; Colom, 2007; Escámez, 2007; García del Dujo, García Carrasco y Asensio, 2007; Gil y Jover, 2007; Sarramona, 2007; Jover, 2006; Jorgensen, 2005; Sing, 2005; CARr, 2004, 2005, 2006, 2007; Wall, 2001; Altarejos, 1998. Y, sin olvidar, algunos de los clásicos: Dewey, 1952; Hirst, 1963, 1983; O'Connor, 1967; SChWab, 1969; Moore, 1980; SCHÖN, 1983; DEARDEN, 1984.

3. Por otro lado, casi todos los manuales, reconocidos como tales, de Teoría de la Educación o Pedagogía publicados en nuestro país suelen contar con un apartado, o varios, dedicado a plantear la relación, integración, dependencia, o como se quiera definir, entre la teoría y la práctica. Valgan como ejemplos más recientes, entre otros, el texto publicado en 2009 por García Aretio, Ruiz Corbella y García Blanco; y el publicado en 2010 por Aznar, Gargallo, Garfella y Cánovas cuyo título es doblemente sugerente: La educación en el pensamiento y la acción. Teoría y praxis.

4. Carr propone también una distribución con dos partes, aunque con sus propias peculiaridades: "Por un lado, están las cuestiones formuladas por la comunidad académica sobre la medida en que la investigación educativa cumple con los criterios epistémicos característicos de la investigación científica social de calidad -por ejemplo, cuestiones relativas a su rigor teórico o a la validez de los conocimientos que genera-. Por otro lado, están las cuestiones formuladas por la comunidad educativa acerca de la medida en que la investigación educativa cumple con los criterios de relevancia práctica -por ejemplo, cuestiones relativas a la contribución de la investigación educativa para la política educativa o la mejora de la práctica educativa $\rightarrow$ (CARR, 2007, 271). 
de formar al profesorado para tener las competencias necesarias que le permitan generar procesos de intervención educativa en torno a la función docente (Touriñán, 1987a, 34-42; 1987b, 82-102), es decir, circunscritos a áreas culturales de aprendizaje, situaciones grupales -grandes o pequeñas-, secuencias de instrucción y evaluación de procesos y resultados.

Otro gran ámbito donde se expresa el problema de la teoría y de la práctica es de carácter epistemológico (Touriñán, 2008; Sáez, 2007; Touriñán y Sáez, 2006). Probablemente sea la línea de trabajo más explotada entre los teóricos de la educación y los aficionados a la epistemología pedagógica. En este grupo el problema de la práctica está relacionado con las disciplinas que generan el conocimiento de la educación. Aquí se incluirían los debates, algunos clásicos, en torno a la relación entre las ciencias de la educación, las ciencias pedagógicas, la pedagogía, la teoría de la educación, la filosofía de la educación, etc. Ahora bien, en este caso no estarían sólo los problemas epistemológicos relacionados con las disciplinas sino también los relativos al alcance teórico y práctico del saber, en general, y, por tanto, del saber de la educación en particular.

Algunos autores, como Labaree, se han referido a las diferentes capacidades que entrarían en juego en los dos ámbitos antes señalados. Con respecto al primero, el referido a la enseñanza, se caracterizaría por ser "normativo", "personal", "particular" y "experiencial". Mientras que las tareas investigadoras se caracterizarían por ser "analíticas", "intelectuales", "universales" y "teóricas". Cada categoría se correspondería con su "contraria" o, si se quiere, "complementaria" (Labaree, 2003, 16-20, cursivas del original)5.

Aunque, como hemos dicho, lo habitual en esta línea de investigación es que se diferencian estos dos tipos de ámbitos o grupos de problemas en torno a la relación de la teoría y la práctica educativa, a nuestro juicio es necesario abrir un tercer grupo o si se quiere un subgrupo del primero. Consideramos que existen unos problemas peculiares, con respecto a la práctica, cuando pensamos en situaciones en las que predomina no la enseñanza de un saber cultural, sino la relación educativa en sí misma. Estamos hablando de situaciones en las que el problema de la práctica se circunscribe a saber elaborar o determinar un juicio educativo acertado para un sujeto. Podemos pensar, por ejemplo, en unos padres aconsejando a sus hijos en una situación familiar. También lo encontramos en la labor de tutoría de un profesor, de un orientador en un centro, de un monitor de campamento de ocio y tiempo libre, de un educador de menores, de un miembro del equipo de tratamiento de una prisión o, en general, cualquier agente, profesional o no,

5. Esta clasificación de capacidades puede ser muy discutida, como veremos, desde ciertos puntos de vista. Sin embargo, es interesante señalar que el trabajo de Labaree, junto con otros, pretende establecer las diferentes capacidades que se requieren cuando los docentes aspiran a pasar de su ejercicio profesional habitual a estudios de grado de especialización, máster o doctorado, con marcado peso en la investigación. Vid. Labaree, 2008; Lagemann, 2008. 
que tiene que valorar y enjuiciar el carácter formador o no de lo ya realizado y de lo susceptible de realizar en el futuro. Wiliam considera así que las situaciones de enseñanza, en concreto, requieren sobre todo - no de un modo exclusivo- un saber técnico. Y la función de ayuda educativa, asesoría o juicio educativo, un «saber prudencial", una "sabiduría práctica" (Wiliam, 2008, 434-436, cursivas del original; vid. Bulterman-Bos, 2008). Por supuesto que para ser un buen profesor se requiere cultivar la praxis aristotélica, pero no es lo mismo tener como problema práctico principal el diseño de una unidad didáctica sobre los minerales de la cuenca del Ebro, que una conversación con una presa para ayudarla a valorar si solicita o no al juez estar con su hijo menor de tres años dentro de la prisión. Ambos son problemas prácticos, pero no son el mismo problema práctico.

El artículo tiene como objetivo analizar los problemas gnoseológicos de fondo de la relación entre la teoría y la práctica, esto es, el segundo ámbito referido anteriormente. Consideramos que los presupuestos más extendidos en la actualidad en torno a la teoría del conocimiento son los que marcan las dificultades principales y particulares de la relación entre la teoría y la práctica educativa. Este análisis nos permitirá, a su vez, comprender los problemas de la relación entre la teoría y la práctica, también en la esfera de la enseñanza-instrucción y en la asesoría educativa.

Las tesis principales del texto son las siguientes: consideramos necesario para el conocimiento educativo recuperar cierta perspectiva gnoseológica de la modernidad, algo criticada desde otros parámetros. Esta recuperación supone valorar la posibilidad de que el sujeto se conciba, en ocasiones, como un agente de conocimiento individual, capaz de situarse frente al mundo, de distanciarse, de dejar en paréntesis, llegado el caso, las experiencias personales, tratando de aspirar a lograr comprensiones intersubjetivamente compartidas y, en otras situaciones, iniciar un disenso público y solitario. Esto supondrá, como segunda tesis, reivindicar un conocimiento teórico fuerte de la pedagogía o teoría de la educación, cuyo valor no tiene que medirse únicamente por sus posibles aplicaciones para la práctica. Esta idea nos llevará a diferenciar, sin complejos, dos modelos de formación, reivindicando sus particularidades de origen y de proyección, así como sus herramientas de trabajo: por un lado, la labor de los profesores y educadores en general y, por otra, la de los investigadores especializados en educación. Finalmente, aplicaremos algunas de estas ideas y, sobre todo, la secuencia de sus argumentos para proponer al final un caso concreto de ruptura de la circularidad deductiva e inductiva y la preeminencia hermenéutica de la pregunta teórica frente a la práctica. Los apartados se corresponden con el orden de las tesis apuntadas.

\section{LA RELACIÓN TEORÍA-PRÁCTICA Y LA EPISTEMOLOGÍA POSTFUNDACIONALISTA}

La epistemología pedagógica ha sido calificada, en nuestro ámbito, de narrativa. Colom ha señalado así que 
[...] la Teoría de la Educación a lo único que puede aspirar es a ser narratividad, es decir, a poder ser considerada como teoría narrativa, que es lo mismo que decir teoría débil, fruto del saber no ontológico y sí aplicativo y pragmático. Además, el saber de la Teoría de la Educación es un saber de compromiso-casual, en función de la realidad sobre la que incidir -y dependiente (de otras ciencias de la educación), por tanto con escasa identidad propia (Colom, 2007, 52; vid. Colom y Rincón, 2007).

No deja de tener interés esta calificación porque las revisiones más actuales de la epistemología clásica, fundacionalista o representacionalista (la que se considera que se inicia con los análisis aristotélicos de la realidad, que continúa o mejor alcanza su cima comprensiva con Descartes y termina extendiéndose a toda la constitución básica de las ciencias a mitad del siglo pasado) apuntan también, en sus análisis respectivos, más a una trayectoria circunstancialmente adaptativa y práctica, narrativa, que a un avance puramente epistemológico, de fundamentación, en la investigación (Arregui, 2004). Las exigencias de rigor empirista, de corte positivista, al ser aplicadas al campo de las ciencias sociales, dejan tantos interrogantes abiertos de todo orden que se necesitan otros modelos epistemológicos alternativos o complementarios de los clásicos. De este modo, la epistemología pedagógica no sería una comprensión parcial, singular, adaptada hacia abajo de nuestro campo de estudio, sino que su peculiaridad calificada como narrativa formaría parte de la categoría amplia de referencia a cualquier tipo de ciencia, por lo menos, del ámbito de las ciencias sociales y las humanidades (Quesada, 2009).

En el ámbito internacional, sin embargo, no es usual referirse a una "epistemología pedagógica de carácter narrativo". Lo más extendido sí es la consideración post- o neofundacionalista (Carr, 2005, 2006) que, ulteriormente, apunta a las mismas consideraciones. En efecto,

lo que el postfundacionalismo enseña es que cualquier perspectiva crítica que tengamos en algunas de nuestras prácticas estará siempre basada en otras de nuestras prácticas; que nuestros supuestos y creencias no pueden ser el objeto de nuestra "teorización práctica", porque proporcionan la condición previa imprescindible a nuestra "teorización práctica" (Carr, 2006, 150).

De este modo, el argumento último es que no cabe elaborar una teoría de la educación con las aspiraciones clásicas de la Ilustración y la modernidad científica. Carr lo explica así en un texto que titula significativamente "Educación sin teoría":

Las razones por las que la teoría educativa no ha podido alcanzar sus metas indicadas, no son porque formular generalizaciones teóricas universales sobre la educación sea una meta compleja, difícil de alcanzar, sino porque es una meta imposible que nunca podrá ser alcanzada. Y no puede alcanzarse porque lejos de ser "universal" o "general" tales generalizaciones teóricas son siempre abstracciones del mundo cambiante de la práctica y son formadas siempre por las mismas características de la práctica -su particularidad y contingencia- que la teoría educativa demanda trascender (Carr, 2006, 147). 
Estas posiciones tratan de oponerse, en realidad, a las tradicionales tesis fundacionalistas de la modernidad científica. Por eso, tal vez convenga recordar, en este momento, los puntos cardinales de la razón moderna, del fundacionalismo epistemológico que, en la propuesta de Charles Taylor, son los siguientes: primero, el sujeto es un ser libre y racional desvinculado del mundo natural y social, independiente de estos mundos; en segundo lugar, la posibilidad instrumental de cambiar o transformar estos mundos al mantener la concepción de un yo puntual que se sitúa frente y visualiza ese mundo. Y, por último, la interpretación de la sociedad desde un punto de vista analítico o atomista explicable en términos de propósitos individuales y morales (Taylor, 1997, 26 y ss.). En definitiva, la modernidad mantenía la convicción de que se podía llegar a la certeza si se usaba la razón con rigor y con sistema, con orden. Y que esa certeza abarcaba tanto las relaciones causales empíricas como la consideración de los bienes morales. Más aún, los principios morales de autoría moral responsable presuponían la posibilidad de conocer la realidad.

Nuestro propósito no estriba, en este momento, en analizar y contraponer ambas tesis gnoseológicas sobre las posibilidades del conocimiento, en general, y del pedagógico en particular. Lo que pretendemos, como se ha indicado, es destacar las diversas formas de relación de la teoría y la práctica educativa, y sus problemas. En este caso, de lo que se trata es de mostrar que algunos de los puntos destacados por Taylor pueden mantenerse con ciertas correcciones o matizaciones y que al hacerlo nos situamos mejor en reconocer la importancia de la teoría educativa.

El empuje epistemológico de corte narrativo o postfundacionalista se caracteriza, entre otros rasgos, por apoyar la sobrevaloración estructural o lingüística de los enunciados (vid. Schatzki, 2001).

El pasado siglo xx descubrió la fascinación del lenguaje. Sea por Heidegger o por Wittgenstein, por el estructuralismo o por la hermenéutica, por las mil derivaciones finales en términos de gramatología o deconstrucción, hemos aprendido a buscar en el lenguaje la forma y figura del pensamiento; y la forma y figura de los mundos que nos constituyen, que nos integran y nos sostienen (Lanceros, 2006, 179).

Desde esta perspectiva ( $c f$. Arregui, 2004) lo que importa es el sentido más que la verdad, y el sentido está en el lenguaje, no en la realidad. Los enunciados falsos también tienen sentido. Todo enunciado contiene en sí mismo un sentido y para determinar este sentido no es necesario atenerse, en todos los casos, ni a los hechos ni a la realidad a la que pueda apuntar ese enunciado. De este modo, el análisis del sentido de las cosas se ciñe al análisis del lenguaje que se utiliza para hablar de esas cosas.

Cuando se afronta la cuestión de la elaboración o construcción del conocimiento en la sociedad actual, se tiende a dar por supuesto que lo que existe es la posibilidad de la experiencia del conocer, más que de la realidad del conocimiento. La filosofía clásica considera el conocimiento como el efecto de la adecuación entre el entendimiento del sujeto consciente y la realidad conocida. [...] A lo más que se podría 
llegar sería a hablar de la experiencia del conocer, lo que equivale a hablar de tantas lecturas de un texto como individuos que tienen experiencia de esa realidad (Vázquez, 2007, 261).

Pero aún hay más. Al quedarse en el lenguaje, en la medida que una de las condiciones de posibilidad pragmática de ese lenguaje es la relación del sujeto con el mundo, el estudio de la realidad remite al estudio del lenguaje y, al mismo tiempo, termina por remitir a las formas de vida como lenguaje. En el lenguaje, desde esta perspectiva, están todas las condiciones o posibilidades de la realidad incluyendo la comprensión y valoración de las formas de vida de interacción del sujeto con el mundo.

Consideramos que la sobrevaloración del lenguaje y, sobre todo, su percepción como medida del conocimiento, puede tener el riesgo de terminar creyendo que se conoce cabalmente una realidad sólo por narrar, explicar, contar o relatar diferentes elementos de esa realidad. De este modo, uno de los problemas de esta perspectiva epistemológica es que puede llegar a confundir el método con el objeto. Otro posible error es llegar a creer e incluso valorar como algo positivo que la realidad puede cambiar si cambiamos el modo de expresarla. Los sofistas vuelven a estar al acecho: la verdad está en decirlo de otro modo, de otra manera, con otras palabras, con otro orden y énfasis.

Una de las tesis de este texto, en relación con la proyección práctica del conocimiento pedagógico, es que no debemos rechazar del todo cierta posibilidad metacultural del logos. Cabe reconocer, más allá de una idiosincrasia particular, de unas relaciones particulares y de un logos local e históricamente situado, la visión de una intuición de una verdad práctica cuando asumimos para siempre la ganancia de esa visión, cuando la afirmamos como deseable para todos. Cuando un educador se negó, en su momento, a seguir poniendo a los alumnos que no rendían, como medio de motivación, unas orejas de burro de cartón grandes y coloreadas, percibió desde dentro de su cultura y marcos referenciales que cabían otras posibilidades, que ésa no era una acción digna para ese alumno, para cualquier alumno y para siempre, se cuente como se cuente, y amanezca el día como amanezca (Gil, 2003a, 88). Al discutir estas ideas, Oancea y Pring señalan:

La investigación, por lo tanto, no puede ser vista como algo que ofrece una base firme desde la que proceder con absoluta confianza, ya sea en la formulación de la política o en la búsqueda de prácticas en particular. Al mismo tiempo, esto no implica que no haya tal cosa como el conocimiento de la investigación -que todo lo que cuente no sean más que construcciones sociales, relativas al punto de vista particular de uno mismo y abierto libremente a las diferentes construcciones sociales. Como vamos a argumentar, la falta de verificación concluyente, y la posibilidad permanente de mejorar aún más las propias conclusiones a la luz de la evidencia, no implica que no haya condiciones de verdad para lo que uno afirma y por lo tanto ninguno modo de falsificar las conclusiones investigadas. El perfeccionamiento de la perspectiva de uno presupone una realidad, 
«EDUCACIÓN CON TEORÍA”. REVISIÓN PEDAGÓGICA DE LAS RELACIONES...

independiente de lo que sepamos, lo que nos fuerza a ser más precisos (Oancea y Pring, 2008, 27) ${ }^{6}$.

Consideramos que cabe perfectamente una aspiración teórica universalista, al tiempo que adaptada a las circunstancias, a los sujetos y a las culturas. Narrar cómo se ha conseguido el voto de la mujer en un país determinado no explica las razones de fondo por las que asumimos como logro justo y adecuado esa conquista, para siempre y en todos los casos. Tampoco nos sirve argumentar que cada país tiene su propia bistoria de la conquista del voto femenino. Ni que las experiencias no pueden ser intercambiables, etc. Buscamos un modo transcultural de razonar y explicar el valor de ese logro y esta búsqueda o aspiración, se termine logrando o no, no es ninguna limitación, todo lo contrario.

Por otra parte, mantenemos que puede tener en ocasiones cierto interés pedagógico concebir la investigación desde la perspectiva de un sujeto quien, en diálogo con otros, se siente individualmente responsable de su saber y de su actuar y, llegado el caso, de romper, de disentir, con su entorno y, hasta donde se pueda, de sus vínculos. Es verdad que no somos una entelequia analítica, individual, desvinculada de nuestro contexto cultural. Es verdad que los seres humanos, la elaboración de su conocimiento, forma parte de una relación interna y externa con la cultura que nos ha tocado vivir y con los horizontes de significado de esa misma cultura. Es verdad que nuestro disenso es parte también de la interpretación posible de nuestra cultura. Pero siendo verdad estas ideas, también lo es que el disenso modifica lo cultural porque no es una proyección mimética de lo ya pensado y hecho, sino porque nos ofrece otras posibilidades imaginadas de fines valiosos que percibimos mejores desde algún punto de vista (Gil, 2008). Pues bien, nuestra propuesta es que esta forma de razonar debe formar parte habitual del estilo y el fondo de los juicios educativos y sus teorías. En el campo educativo, la aplicabilidad teórica de una idea o de una propuesta requiere, en ocasiones, dejar en paréntesis, que no anular ni desconsiderar, el entrelazado cultural y relacional de los sujetos con esa cultura. En algunas ocasiones el mejor modo de pensar a un sujeto en sus posibilidades educativas y transformadores radica, precisamente, haciéndolo al contrario y en resistencia con lo que nos dicen todos los datos de su situación. Hacer el esfuerzo intelectual de que podemos razonar mirando el

6. Este artículo, junto con otros, constituye uno de los últimos números monográficos de la Journal of Philosophy of Education dedicado al tema de la investigación educativa y la relación entre la teoría y la práctica. Los artículos recogidos en esta revista son, entre otros, los que se presentaron en un influyente seminario titulado "Las bases epistemológicas de los hallazgos de la investigación educativa", organizado por el Teaching and Learning Research Programme (TLRP) del ESRC (Economic and Social Research Council). Los ponentes asistentes a este seminario fueron: David Bridges, James Conroy, Robert Davis, John Elliott, Morwenna Griffths, Penny Enslin, Lorraine Foreman Peck, Dominik Lukesô, Gale Macleod, Jane Murray, Alis Oancea, Richard Pring, Lesley Saunders, Paul Smeyers, Richard Smith Michael Watts. 
margen exterior, extraño e imaginado de nuestro propio lado, que podemos y, en muchos casos, debemos, plantear soluciones a los sujetos desde fuera de su situación relacional, para comprenderlos mejor, para poderles comprender más allá de su propia situación y lenguaje.

¿Por qué no vamos a ser capaces de mantener un difícil y delicado equilibrio entre el saber local, histórico y culturalmente arraigado con el saber que aspira a algo más? Sólo puedo ser humano desde una interpretación concreta. Como saber teórico, es siempre una interpretación parcial; sin embargo, como saber práctico, vital, para mí, es la interpretación total. Ya nos enseñó Geertz (1992, 118-130) que en todas las culturas podemos encontrar empíricamente la expresión de la vinculación entre lo que las cosas son y lo que deben ser, entre la ontología y la ética, pues en toda cultura las normas éticas se fundamentan en una idea de la naturaleza de las cosas; idea que es, a su vez, interna a esa misma cultura. Toda cultura tiende a fundamentar de modo absoluto sus propias verdades no sólo por un afán etnocentrista, sino porque ninguna cultura se contentaría con fundar relativistamente sus propias normas. La verdad no es algo probablemente diferente, tal vez sea el sentido último de esa aspiración de fundar lo particular, para llegar así a lo más común.

Consideramos que la teoría de la educación debe aspirar a mejorar, desde algún punto de vista, el orden de lo culturalmente sabido y valorado ( $c f$. Rodríguez Neira, 2002, 434 y ss.). Si la epistemología pedagógica pretende tener cierto carácter científico ha de seguir caracterizándose por mantener, dentro de sus propias posibilidades y límites, cierta aspiración metacultural. Esta propuesta nada tiene que ver con un absolutismo axiológico ni con la ruptura historicista ni, por último, con un aislamiento del yo. Somos y nos educamos en cambios de estado deseables en términos de relación con el mundo y con los demás. Sin esa relación no hay cambio, ni educación, ni sujeto. No estamos, por tanto, reivindicando una vuelta a un representacionismo-espejo. Nuestra tesis se centra en considerar que las propuestas prácticas basadas en una teoría educativa tienen que insertarse y concretarse específicamente en la singularidad del espacio y el tiempo del sujeto, pero que la ocurrencia en torno al valor de esa práctica tiene, en sí misma, una aspiración a trascender, desde algún punto de vista, el sentido y posibilidades de ese sujeto, de ese tiempo y de ese espacio.

Se ha criticado mucho el afán universalista de la modernidad. Pero en realidad el problema del conocimiento pedagógico y de su relación con la práctica, con el valor de la práctica, no se plantea correctamente si de lo que se trata es de resolver una dicotomía o alternativa entre quienes consideran que es posible un saber absoluto o que sólo existe un saber históricamente contextualizado. El proceso educativo, como se ha indicado, es un proceso de cambio deseable, en situaciones espaciales y temporales concretas, y casi siempre debido o causado por una situación de relación. Educar consiste en ayudar a mejorar a un sujeto. Educar consiste en iluminar la experiencia de posibilidades de mejora que tenemos de nosotros mismos. Por tanto, la inserción cultural del conocimiento pedagógico tiene que 
aspirar, precisamente, por atenerse a su finalidad, que acabamos de señalar, a reflexionar sobre otras experiencias, posibilidades, interpretaciones, del mundo y de lo humano. Y para lograr el descubrimiento de esas otras posibilidades no queda más remedio que establecer una distancia imaginada con respecto al tiempo, al espacio y a los fines dominantes. El universalismo de la modernidad necesita pues muchas correcciones, adaptaciones y, tal vez, menos arrogancias e intolerancias, pero tampoco ha de verse, en cualquier caso, como un callejón sin salida para la teoría y la práctica educativa. Más bien es lo que nos permite, posibilita y empuja, como aspiración, a encontrar lo mejor para el hombre entre lo no realizado ni tan siquiera pensado en la cultura actual.

\section{LA RELACIÓN DE LA TEORÍA Y LA PRÁCTICA EDUCATIVA EN LA INVESTIGACIÓN PEDAGÓGICA}

Como hemos mostrado, algunas de las tesis de las posiciones epistemológicas postfundacionalistas nos pueden llevar a desconsiderar, en exceso, la teoría educativa frente a la práctica. De hecho, la propuesta del propio Carr hay que entenderla así, pues su "educación sin teoría", que veíamos antes, no debe contemplarse como una posición deseable en sí misma, sino como una consecuencia del descrédito continuado con respecto a la estabilidad de ciertos marcos de referencia interpretativos. Esta tendencia generalizada en las ciencias sociales tiene, a nuestro modo de ver, varias manifestaciones particulares en la investigación pedagógica. Por un lado, se ha pasado a reivindicar casi como único criterio de validez epistemológico el conocimiento pedagógico que tenga una aplicación directa, o lo más directa posible, en la práctica. Por otro, se tiende a reducir la práctica, en la misma línea de desacreditación de la teoría, a las situaciones instructivas de aprendizajes culturales, sin incluir, con igual valor de certeza, e incluso excluyendo de las posibilidades del saber, los juicios educativos sobre los diversos modos de conducirse en torno a los fines de la vida (vid. Barrio Maestre, 2008). Otra consecuencia es que se mezcla, cada vez con más mezcolanza, la teoría educativa del que enseña y, según el caso, educa, con la del investigador en Teoría de la Educación. Vamos a analizar estos argumentos.

Gran parte de la epistemología pedagógica elaborada en nuestro país, que surgía de nosotros mismos como cultivadores de la Teoría de la Educación, centraba su máximo interés en reclamar que sirviese para la práctica. Tal vez lo que nos ocurría, entre otras acertadas razones, ya apuntadas, de carácter histórico-ideológico, o precisamente por ellas ( $c f$. Jover, 2003; Colom y Rincón, 2004, 38 y 41; Jover y Thoilliez, 2010, 61) es que tratábamos de ganar identidad como especialistas prácticos de la enseñanza y la educación ${ }^{7}$. Es lo que Ruitenberg, al referirse a la

7. Esta cuestión tiene más ramificaciones de las que podemos tratar aquí. La reivindicación práctica adoptó, inicialmente, a nuestro juicio, una muy acertada y necesaria perspectiva tecnológica 
investigación teórica de la educación, denomina "el estado de ansiedad de la academia" (2009, 316). A nuestro juicio, la función de la investigación en Teoría de la Educación no tiene ahí su única ni, tal vez, principal finalidad.

Hacer Teoría de la Educación o Pedagogía, desde una perspectiva epistemológica, consistía en afirmar que debíamos hacer una teoría práctica de la ciencia de educar. De este modo, a nuestro juicio, no se hacía realmente teoría educativa sino que se demandaba que se hiciese una teoría práctica. Y no es lo mismo. Entiéndase bien, no estamos diciendo que nos hemos limitado a reivindicar que se haga algo que no se hacía. Estamos diciendo que, para animar a que se hiciese investigación pedagógica que sirviese para la práctica educativa, hemos concentrado nuestros esfuerzos en reivindicar que se elaboren teorías prácticas. Lo diremos de otro modo. La teoría estrictamente nunca dice nada sobre la acción, la práctica o los cursos de acción. Si una teoría de las que llamamos educativa dice algo de la acción, la práctica o los cursos de acción no es por ser teoría sino por tener como objeto a la educación. Y si no lo dice no significa que sea una mala teoría ni que no sea una teoría educativa, es que probablemente no está concentrando la atención en la educación como una acción.

Necesitamos teorías educativas, reflexivas, abiertas y claras que nos ayuden a determinar qué es humanamente valioso para la formación de un sujeto. Estas teorías no son buenas por ser aplicables -técnicamente o no-; son buenas porque se corresponden con lo que consideramos que es valioso para el ser humano en desarrollo. El ajuste técnico de un fin es una condición de aplicación del fin -una de las muchas que puede tener-, pero no es una condición del conocimiento del fin como humanamente deseable. El que algo me haga llegar a una meta no otorga valor a esa meta. Como hemos señalado, debemos concentrarnos, no exclusivamente, pero sí de modo relevante, en las tareas teóricas de explicar, comprender y sintetizar la realidad educativa.

Pensar la educación en toda su complejidad implica aprender a proyectar un interés pedagógico, amplio y general, por saber desentrañar las múltiples vías de acceso a la realidad educativa, por adoptar diversos criterios de racionalidad, por aceptar una pluralidad de niveles de fundamentación teórica y por acoger orientaciones para la acción práctica de diferentes niveles y hasta estilos (Gil, 2003b; Gil

que al aplicarse, curiosamente, a los tradicionales fines y valores educativos (EsCÁmEz, 2007) amplió, también acertadamente, sus enfoques a miradas más experienciales, dialógicas y críticas. Toda esta trayectoria que, en realidad, debió verse siempre sólo como una tarea pendiente, responsable y equilibradora de nuestra agenda de trabajo, se usó, sin embargo, como metadiscurso identitario de una única Teoría de la Educación práctica-tecnológica. Pasamos así de la cosificación y dependencia disciplinaria de carácter filosófico, a otra cosificación de "café para todos" (GiL, 2003b). Esta etapa ha sido relativamente superada, lo que tal vez nos permita acentuar y equilibrar ahora, con las correcciones necesarias y ya aprendidas, las tareas teóricas de explicar, comprender y sintetizar la realidad educativa (toda la realidad educativa) con los necesarios conocimientos de transformación y aplicación (todas las formas posibles de transformar y aplicar). 
y Jover, 2007). Como apunta el profesor Trilla (2005) en su texto "Hacer Pedagogía hoy", si pretendemos responder a las necesidades del mundo educativo, tenemos que defender una concepción orientadora de la práctica más plural, con diferentes rangos, desde las sugerencias más experienciales a la normatividad más tecnológica (Trilla, 2005, 295 y ss.; cf. Martínez, 2005, 671 y ss.; cf. González Faraco y Gramigna, 2009, 84).

Pero también creo que debemos defender una elaboración teórica de la educación más plural, tenga en principio aplicabilidad práctica o no. No estamos de acuerdo, en todos los casos, como criterio de demarcación, en que "la investigación podría ser más útil si su estructura y organización estuviesen mucho mejor vinculadas a las necesidades prácticas del sistema educativo" (Burkhardt y Schoenfeld, $2003,3)^{8}$. No parece acertado limitar la elaboración de la Teoría de la Educación a sus posibilidades prácticas porque no siempre podemos pensar con claridad una correspondencia lineal o causal entre la teoría y la práctica. Es decir, no podemos saber de antemano cuándo una teoría, un argumento, una idea, tiene aplicación práctica o no. Poder diferenciar este hecho supondría tener un conocimiento claro de los límites de la teoría y un conocimiento claro de los límites de la práctica. Y no lo tenemos, no lo sabemos y, sobre todo, no podemos saberlo. Como nos recuerda Labaree "[...] la investigación que parece como un ejercicio abstracto en la construcción de teoría, en un tiempo y lugar puede llegar a ser muy relevante para los propósitos prácticos que no fueron considerados en el momento en que se realizó la investigación" (2008, 423).

No sabemos de antemano e inmediatamente el alcance práctico de nuestras teorías. No tenemos un procedimiento mecánico para comprobarlo. Podemos perder horizonte y hondura investigadora si adoptamos entonces como estrategia seleccionar sólo los temas y métodos que contengan en sí mismos condiciones de aplicabilidad práctica inmediata. La función principal de la investigación pedagógica es explicar, comprender y sintetizar claves interpretativas de carácter educativo. La Teoría de la Educación debe considerar que entre el conocimiento que obtenemos de los diferentes saberes y su racionalidad práctica (tecnológica o experiencial) hay un campo específico de validación pedagógica y que esta validación o reflexión es de carácter teórico. "En este sentido, y como tal teoría, pretende una fundamentación que sea válida para cualquier teoría particular o específica [...]" (Colom, 2007, 55). La pedagogía adquiere identidad como tal por la consistencia teórica de sus ideas, no por la aplicabilidad de las mismas.

8. Estos autores se refieren a la necesidad de un enfoque o aproximación ingenieril o de ingeniería. Esta categoría hay que entenderla de un modo amplio: "La aproximación "de ingeniería" de la investigación está directamente relacionada con su impacto práctico -comprender cómo el mundo trabaja y ayudarlo a trabajar mejor, diseñando y desarrollando sistemáticamente soluciones de alta calidad para los problemas prácticos. Se construye desde otras aportaciones de la investigación, en la medida que sea posible, y yendo más allá de ellas mismas" (BurkHardt y SCHOENFeld, 2003, 5). 
La Pedagogía se pedagogiza, la Teoría de la Educación se sustancializa más y mejor cuanto más esfuerzos de explicación, comprensión, síntesis y sistematización realice de las diferentes sensibilidades sociales e investigaciones científicas acerca lo que sea valioso en la formación de los sujetos.

Por eso, consideramos necesario diferenciar, en nuestras agendas de trabajo, con la prudencia debida y sin posiciones extremas, ni excluyentes, ni juicios valorativos o jerárquicos, la investigación educativa centrada en la enseñanza y la ayuda educativa, de la investigación educativa generadora de conocimiento general sobre la educación como experiencia personal y cultural ${ }^{9}$. "Al identificar educación con enseñanza-aprendizaje planificada en organizaciones institucionales, la práctica se asimila con el quehacer de los profesores; en consecuencia, la legítima teoría de la educación, aquella que beneficia y transforma la práctica, es la que genera la reflexión desde la propia práctica; los no partícipes de esa práctica padecerán de ilegitimidad teórica" (García Carrasco y García del Dujo, 2001, 29). Ambas investigaciones tienen requisitos y objetivos diferentes, y plantean relaciones con la práctica diferentes. La primera es una teoría interesada, desarrollada y proyectada en la práctica. La segunda, por el contrario, no tiene que ser práctica, sino una buena teoría ( $c f$. Hammersley, 2008, 6; 2005, 7; Oancea y Furlong, 2007; Oancea, 2005, 2007). Como argumenta Labaree,

(1)os profesores están ocupados en primer lugar con la práctica de la mejora social, se apoyan en las relaciones personales con los estudiantes situados en un tiempo y en un lugar, y el conocimiento profesional que construyen es en gran medida una acumulación de experiencias clínicas. Los investigadores en educación están ocupados en primer lugar con una práctica de análisis social, se apoyan en las concepciones intelectuales de la educación generalizadas en distintos contextos, y el conocimiento profesional que construyen es en gran medida una red de teorías. Las condiciones diferentes de trabajo llevan a diferentes modos de práctica profesional y a diferentes modos de pensar sobre la educación (Labaree, 2008, 421; vid.: Bulterman-Boss, 2008, 412; Clemente, 2007, 27; Romero, 2006, $106-109)^{10}$.

Brezinka nos recuerda, por su parte, que «el llamado saber pedagógico estrictamente científico es, por un lado, impreciso en sus límites, inseguro y, por principio, incompleto; y, por otro lado, se halla muy limitado en sus aplicaciones prácticas y posee un contenido poco relevante» (Brezinka, 2002, 407). La Teoría de

9. Son interesantes las apreciaciones de Kemis en torno a lo que denomina "arquitecturas de la práctica", "metaprácticas" y "ecología de las prácticas", para hacer referencia al aprendizaje de las experiencias prácticas entre diferentes profesionales y agentes ocupados en la educación y la interrelación entre todos ellos (Kemis, 2009, 3).

10. Son muy interesantes los argumentos que utiliza LABAREe para criticar el debate internacional de la relevancia de la investigación educativa. "La relevancia -dice- es el ojo del espectador" (2008, 422). 
la Educación debe mantenerse siempre en una perspectiva abierta, integradora y sintetizadora porque hay espacios de reflexión y actuación, así como retos de conocimiento o preocupación en las líneas más vanguardistas de las ciencias sociales, que nos deben situar en un orden de conocimiento teórico-práctico amplio e integrador que nos permita participar de todas las posibles preocupaciones formativas del hombre (cf. Gil, 2003b; Gil y Jover, 2007).

La pedagogía adquiere, pues, un claro compromiso teórico, junto al indiscutible compromiso práctico. El compromiso teórico no puede reducirse al de la investigación y la reflexión en la acción, sino que ha de ampliarse a la investigación y la reflexión sobre los componentes, contenidos y valores clave de la cultura (García del Dujo, García Carrasco y Asensio, 2007, 178).

Para lograr constituirnos en un saber realmente abierto y sintetizador de lo que se piensa e investiga en todos los saberes científicos y humanísticos, y de lo que se "mueve" dentro de las dinámicas culturales y sociales, no podemos renunciar a ningún nivel de especulación, ni a ningún modo de llegar a la práctica. Tenemos que acostumbrarnos a "[...] mirar la riqueza de las tradiciones intelectuales que pueden contribuir a la comprensión educativa como una fuente de fascinación antes que de frustración, como algo para celebrar antes que para lamentar" (Bridges, Smeyers y Smith, 2008, 6). Es necesario ampliar nuestros modos de conocer la educación "[...] atender a formas de investigar que son "sistemáticamente" excluidas por la estrechez [...]" de sus condiciones de investigación (Smith, 2008, 196) ${ }^{11}$.

Para aceptar estas ideas es conveniente diferenciar dos aspectos muy relacionados pero diferentes. La Teoría de la Educación es la ciencia de la educación, pero la fuente de conocimiento pedagógico es, en casi todos los casos, el propio sujeto en relación consigo mismo y con la realidad cultural que le rodea. El deseable rigor al que debe aspirar la Teoría de la Educación no debe suponer, entonces, la minusvaloración de un dato radicalmente importante: lo que un hombre hace o decide hacer para educarse a lo largo de su vida no es una decisión científica, ni técnica sobre su formación, sino unas deliberaciones entre deseos, gustos, ideas, actitudes, etc. El criterio de valor pedagógico no puede residir, por tanto, sólo en el cumplimiento de directrices epistemológicas internas a la Teoría de la Educación, sino aspirar a aceptar múltiples vías de comprensión de lo educativo, independientemente de que no siempre podamos alejarnos de la

11. Smith cita los estudio de casos, los relatos personales, la investigación-acción, la filosofía y lo que se ha denominado el "giro romántico", que podría resumirse en la siguiente forma de proceder: "En lugar de conocer el mundo deberíamos estar en sintonía con él, ser sensibles a él. Deberíamos "resonar" con él, compartir sus ritmos -el modo en que podríamos estar con el mundo natural si nosotros mismos nos abriésemos en vez de analizarlo, reduciéndolo a sus elementos constituyentes [...]" (SMITH, 2008, 186). 
dependencia disciplinar o de que no podamos analizarlas desde el rigor científico y tecnológico dominante.

"Si la educación es un complejo y multifacético fenómeno social que tiene lugar en una variedad de instituciones y situaciones, entonces tiene que ser estudiada de muchas maneras diferentes" (Lagemann, 2008, 424). La educación es, antes que el objeto de una ciencia, una realidad social y una aspiración humana. Los enfoques epistemológicos cambian, no así la presencia cultural de la educación, con una forma o con otra. Para nosotros, es importante, en consecuencia, que cualquier propuesta epistemológica tenga en cuenta, de algún modo, ese dinamismo social y científico.

Pero dicho esto pienso que también debemos aprender a compaginar, y no veo contradicción alguna, este escenario con estudios con un estilo pedagógico más definido. Por estilo pedagógico más definido entiendo la preocupación y el interés por referirnos al mundo educativo o por construir elementos que tengan interés para el mundo educativo. "Abandonar la investigación en la educación en favor de la investigación educativa" (Smeyers, 2010, 114). El problema de la Teoría de la Educación no es su excesiva especulación. El problema de la Teoría de la Educación no es tampoco su excesivo afán práctico, tecnológico o científico. El problema, a nuestro juicio, de la Teoría de la Educación es que en muchas ocasiones no hablamos de educación. Y si no hablamos de educación, lo mismo da ya que hagamos teoría o práctica. Como ha ironizado Smeyers, lo que importa es el contenido, no sólo el método, hacer cosas interesantes y "desenredar lo que está involucrado en los casos particulares de la práctica educativa" (Smeyers, 2010, 113 y 114). En definitiva, no se trata tanto de pensar que hacemos educación por atender a la práctica, como de adoptar siempre una perspectiva educativa de la realidad. Somos pedagogos y pedagogas no por ser prácticos ni por ser teóricos, sino por colorear de educación al mundo y a las personas.

No quiero transmitir una imagen de que abrazo cualquier tipo de teoría de la educación, ya sea como disciplina universitaria o como ejercicio intelectual. No, en ningún caso. Es verdad que es difícil, en muchas ocasiones, mantenerse en lo que se ha defendido hasta aquí, pues el número de tonterías con ropaje pedagógico no deja de crecer. Pero hay que evitar la arrogancia teórica frente al futuro de lo desconocido. Nadie dispone hoy de un bisturí perfectamente afilado para saber delimitar, cortando, cuál es el futuro normativo de la educación. Es decir, qué cosas pasarán y cuáles no, y por qué. En cientos de laboratorios del mundo, se desechan diariamente otras tantas tomas, pruebas, análisis, intentos vanos de apuestas por el conocimiento, ¿por qué no íbamos a aceptar lo mismo en la teoría pedagógica? Considero, no obstante, que la pluralidad de temas, intereses y métodos tiene la ventaja, precisamente, de ir comprobando cuáles son, entre todas las posibles, las mejores modalidades de teorizar tanto cuando buscamos incrementar el saber pedagógico en general, como cuando lo que pretendemos es mejorar la formación del profesorado y los procesos de enseñanza y asesoría educativa. 
4. LA RELACIÓN DE LA TEORÍA Y LA PRÁCTICA EDUCATIVA EN LA ENSEÑANZA Y LA EDUCACIÓN. Acerca de lo razonable. El caso de MatThew

El teórico de la educación Max Van Manen nos cuenta el siguiente caso:

Matthew es un alumno difícil y no está dispuesto a hacer un esfuerzo en la escuela. La tutora de Matthew se siente responsable. Tiene que hacer algo con él. ¿¿Sobre qué base debería o podría actuar de manera claramente pedagógica? Se pueden pedir datos acerca de su vida a la psicóloga de la escuela o de la asistente social. Por ejemplo, Matthew proviene de una familia muy desfavorecida. Tiene una baja autoestima académica. Su habilidad lectora no es la que correspondería. Los padres de Matthew muestran muy poco interés por su éxito o por su fracaso escolar. Sin embargo, los profesores creen que Matthew es inteligente. Los exámenes demuestran que está rindiendo por debajo de sus posibilidades. Generalmente, sobre la base de un diagnóstico de información empírica como éste, se pueden deducir algunas pautas de actuación. La psicóloga de la escuela podría recomendar un programa de asesoría para Matthew. El director del centro podría recomendar algún sistema para hacer un seguimiento de la asistencia a clase por parte de Matthew. El asistente social podría sugerir una serie de visitas a la familia. La tutora está preocupada porque Matthew falta mucho a clase y puede que abandone pronto los estudios. Ella cree que debería continuar y pasar a la secundaria y aumentar sus posibilidades en la vida. Pero algunos de sus compañeros de la escuela argumentan que no se debe tratar de que los adolescentes permanezcan en ella contra su voluntad.

¿Qué debe hacer la profesora? Hoy tiene clase con Matthew. ¿Debería pedirle que tuvieran una charla después de clase? ¿Qué debería decirle? ¿Qué resultaría más adecuado en esta situación? ¿Cómo puede la profesora involucrar a Matthew? ¿Cómo debe acercarse a él?

Tanto el conocimiento empírico como los principios éticos y morales son importantes en la pedagogía; de hecho, no se pueden pasar sin ellos. Sin embargo, tampoco son la esencia de la pedagogía. El momento pedagógico es la respuesta concreta y práctica a la pregunta ¿qué hacer en este momento? La esencia de la pedagogía es tener una respuesta en el trato con el niño en cada situación específica. La pregunta "¿Qué hacer?" no deriva directamente de los "hechos" (Manen, 1998, 59-60).

Es cierto que nuestras decisiones deben basarse en un sabio equilibrio entre lo que la realidad nos muestra y las categorías que usamos para valorar esa realidad. También lo es que tenemos que adaptarnos, en su caso, a las formas de valorar la realidad desde las tendencias culturales, bien generales, bien con respecto a algún tipo de tradición e incluso, podría ser, desde una ocurrencia única de la tutora. De igual modo, es correcto reconocer que no debemos adoptar una decisión recurriendo a un ideal tan alejado de las posibilidades reales de Matthew que impida a éste su consecución. En cualquier caso, ya sea que asumamos estos o cualquier otro argumento previo, proporcionado por el conocimiento acumulado desde la investigación pedagógica, en rigor, bay que aceptar la primacía y la preeminencia cognoscitiva, valorativa y ética de la teoría. La realidad sólo tiene la voz que nosotros le ponemos. 
La práctica sólo se realiza en los sentidos teóricos que le atribuimos. No hay nada en el caso de Matthew que nos diga qué tenemos que hacer. Lo que finalmente decidamos hacer es una decisión nuestra, apoyada o no, de un modo fuerte o suave, en los datos de la realidad. Datos que, a su vez, han sido interpretados bien por la tutora, otros profesionales, el propio Manen, el traductor si se quiere, o por nosotros mismos leyendo el caso expuesto.

Si, tras la decisión adoptada por la tutora, el rendimiento de Matthew aumenta, así como mejora su comportamiento en clase, diremos que ha acertado, que ha dado con una verdad práctica para Matthew, sólo, en este caso, para él y en esta situación. Pues bien, lo que me interesa resaltar en este momento es que esa verdad práctica que, como tal, se expresa en lo operable -en la situación concreta y el sujeto concreto-, no se extrae directamente ni de la situación o contexto ni tampoco del propio Matthew. En definitiva, las circunstancias concretas, los sujetos concretos, las aulas determinadas, etc., ni nos dan el fin teórico, ni tampoco la voluntad de su actualización práctica. Por supuesto que la pericia, experiencia, grado de experto que tenga la tutora influye decisivamente en el proceso de decisión. La tutora podrá ver más variables, integrarlas, imaginar más situaciones de futuro, mostrar más velocidad en su juicio, diferenciar lo relevante de lo secundario, etc., pero ni Matthew ni los informes le siguen diciendo qué tiene que hacer. Cuando se dice atender a la realidad se está queriendo decir que cambiemos el modo de entender teóricamente esa realidad, que recurramos a otras categorías, que observemos sin prejuicios cómo se modifica la realidad por la aplicación de nuestras ideas, que seamos capaces de pensar y entender otros estilos de vida, otro modo de ver a Matthew, otros lenguajes.

\subsection{Ruptura del círculo interpretativo. La toma de decisiones para la práctica educativa}

¿Cómo se vincula, entonces, la teoría con la práctica? ¿Cómo determinan el profesor y el educador la oportunidad del momento? ¿Cómo se reintegran e interpretan todas las variables reconocidas? ¿Qué hacemos pues con Matthew?

Los metodólogos de las ciencias sociales, tanto si defienden el individualismo como el holismo metodológico (Elster, 1996, 20 y ss.), nos aconsejarán establecer una cadena, un "mecanismo" ${ }^{12}$ de decisión de carácter deductivo e inductivo hasta llegar a la expresión mínima o básica, respectivamente, y llevarla así a la práctica. Considero, por el contrario, que una de las peculiaridades del pensamiento pedagógico estriba, precisamente, en saber parar esa cadena de decisión por una buena

12. "Los mecanismos -explica Lago- muestran cómo los valores de una variable independiente influyen en las oportunidades, las creencias y/o los intereses de los actores, y cómo las acciones e interacciones de estos generan los valores de la variable dependiente" (LAGO, 2008, 63-64). 
razón, por un buen motivo. En algún momento, la cadena deductiva o inductiva acerca de qué hacer con Matthew se para y no por falta de razones, informaciones, opiniones, consejos, sugerencias, datos, investigaciones, informes, valoraciones, etc., sino por la apropiación sobrevenida y acentuada de un motivo. Adoptamos una decisión basándonos en uno o varios motivos, motivos que no tienen que formar parte de la misma cadena inductiva o deductiva de razones o causas de la situación o escenario sino de un argumento sobrevenido que pondera ciertas variables frente a otras. Pues bien, el motivo, el punto de vista, que sobreviene y rompe la cadena deductiva e inductiva es, en rigor, una interpretación, una hermenéutica, una teoría descriptiva y valorativa de la acción imaginada como deseable, como educativa, y una hermenéutica, una teoría descriptiva y valorativa, del sujeto imaginado como educado. El motivo y el argumento que sostiene la decisión es una teoría circunscrita a lo operable o aplicable, en el caso de Matthew, pero se basa y necesita una teoría fuerte centrada en las ideas en torno a lo que consideramos como educativo y como sujeto educado. Carr nos ha vuelto a recordar que se trata de «[...] desarrollar una capacidad de ver las particularidades de una situación práctica concreta a la luz de su significado educativo general, y sobre la base del razonamiento práctico elaborar un juicio informado acerca de lo que sería apropiado educativamente hacer" (Carr, 2007, 277).

En el ámbito educativo se establece la singularidad de que, sean cuales sean las sumas, multiplicaciones y mutuas implicaciones de los fenómenos políticos, sociales y culturales que afectan a las decisiones de los sujetos, cabe considerar que cuando nos encontramos en una situación, escenario u oportunidad educativa, el educador toma de forma individual, ulteriormente, una decisión, jerarquiza los motivos, asumiendo, rechazando o ponderando las variables políticas, sociales, culturales, familiares o escolares que se le pongan por delante. De este modo el individualismo metodológico en pedagogía requiere mantener, como mostramos en el segundo apartado del artículo, ciertas condiciones de la epistemología moderna frente al empuje del postfundacionalismo.

¿Cómo surge, entonces, el motivo de la tutora de Matthew? ¿Cómo aparece o se desvela el punto de vista? Entre todo el universo de lo educativamente deseable, ¿cómo nos decidimos por una acción y no por otra? En fin, ¿cómo se conectan ulteriormente la teoría y la práctica? La tutora actuará de un modo o de otro en función de lo que constituye el universo habitual de sus interrogantes en torno a la educación. Lo que conecta la teoría con la práctica educativa, los motivos o puntos de vista con las acciones, es el reconocimiento de la pregunta que toda acción o experiencia educativa lleva inserta. La pregunta es la primacía de la hermenéutica (Gadamer, 1977). La acción o las acciones que realiza un sujeto han de ser vistas desde la pregunta o las preguntas que el educador se hace. Es esencial a toda acción o experiencia educativa el reconocimiento de la pregunta que lleva inserta. Una experiencia sin pregunta es una experiencia sin sentido. Vivir la experiencia es vivir en la pregunta que la contiene. La tutora de Matthew tiene que vivir en las preguntas de las experiencias educativas. La tutora responde por Matthew las 
preguntas que éste no se hace todavía. Y las preguntas dependen de las convicciones teóricas de la tutora sobre la educación y la vida.

La casuística acerca de las preguntas que llevan insertas las experiencias educativas es interminable. Hay, sin embargo, una metapregunta que da sentido a todas las demás, por la que la tutora debería luchar al inicio y al final de su decisión. Es una pregunta estrictamente pedagógica. La pregunta educativa por excelencia inserta en la acción es una pregunta que busca como respuesta el sí puedo: leer, escribir, analizar, calcular, correr, memorizar, amar, ayudar, resolver, etc. El buen educador, el buen profesor, el buen teórico en educación es el que logra que la acción práctica de sus alumnos sea la respuesta a esa pregunta. La fortaleza teórica del que propone una acción práctica estriba en la capacidad de ensanchar las posibilidades de acción de los sujetos. Lo que nos mueve a realizar una acción en educación, lo singular de la práctica educativa, es la expectativa de lograr que el sujeto o los sujetos hagan algo. Relacionar bien la teoría con la práctica en educación no es ni más ni menos que establecer motivos, puntos de vista, razones y preguntas dirigidas a pensar en los sujetos, a pensar en que Matthew si va a ser capaz de hacer y de llegar a ser lo que los demás no creen.

El modo en que los profesores y educadores puedan mejorar su práctica educativa estriba en que quieran mejorar los motivos de sus decisiones frente la pluralidad de variables que intervienen. Y el modo de mejorar los motivos es ampliar las teorias que les sostienen, es decir, mejorar las fuentes, de todo tipo, que han colaborado a su elaboración y mantenimiento. Por eso nos referíamos antes a las convicciones pedagógicas (Ibáñez-Martín, 2007), son los motivos teóricos fuertes, las creencias fundamentadas (aptas dicen los epistemólogos) las que paran el círculo de decisión para proyectarlo en la práctica. Ernest Sosa nos propone "otra forma en que la causalidad se relaciona estrechamente con la normatividad [...] según conocemos más amplia, profunda, y seguramente nuestras fuentes, inclusive las más básicas, y su fiabilidad, mejor conocimiento pueden constituir las creencias que recibimos de esas mismas fuentes" (Sosa, 2009, 122).

Es cierto que en el campo educativo los elementos aleatorios, las variables sobrevenidas e inesperadas son tan numerosas, con intervención de tantos agentes y secuencias tan imprevisibles, que es reconocido por todos que, salvo determinadas áreas de investigación muy concretas, predomina más la incertidumbre (Bárcena, 2005, 154 y ss.) que la presencia nomológica de unas variables. Sin embargo, frente a la dificultad de elaborar explicaciones sistemáticas, no nos debemos contentar con cualquier comprensión. En la investigación teórica de la educación y en su aplicación práctica debemos permanentemente aspirar, como expectativa, a disminuir el espacio de lo aleatorio, no explicable, comprensible o espurio, por una explicación más sistemática y, si no es posible, por una comprensión razonable, por unos motivos sensatos, asumibles y consensuadamente compartidos.

De este modo, lo que la relación entre la teoría y la práctica precisa con respecto a la función docente y educativa es incrementar la consistencia teórica de las convicciones que sostienen los motivos. La consistencia teórica no garantiza por sí 
misma la eficacia de la acción práctica realizada, sino la persuasión argumental del fundamento del motivo. Es decir, lo que la mejora teórica nos puede proporcionar es la justificación de la ruptura deductiva o inductiva en un punto, no necesariamente la certeza de que Matthew vaya a hacer lo que deseamos. La relación entre teoría y práctica educativa es una relación acerca del sentido razonable de lo que se dice y se hace, y de los motivos que aducimos para romper la circularidad deductiva o inductiva. Por eso necesitamos, como profesores y educadores, más teoría, más justificación reflexiva y especulativa sobre el sentido educativo del motivo adoptado, porque la justificación es una propiedad normativa o directiva del propio motivo, no de la acción práctica. Esta distinción es importante. Una vez adoptada la decisión y propuesta al sujeto, sugerida, invitada, no cabe más que esperar, tener la expectativa, la esperanza de su realización. Pero esto es ya injustificable desde la teoría pedagógica, pertenece sólo al sujeto. Querer entrar en esa decisión supondría sustituir la invitación por la coacción.

\section{REFERENCIAS BIBLIOGRÁFICAS}

Altarejos, F. (1998) Educación y saberes, en Aa.vv. Filosofía de la educación. Temas. Madrid, Dykinson, 175-192.

Arregui, J. V. (2004) La pluralidad de la razón. Madrid, Síntesis.

Aznar, P.; Gargallo, B.; Garfella, P. y Cánovas, P. (2010) La educación en el pensamiento y la acción. Teoría y praxis. Valencia, Tirant Lo Blanch.

BALL, D. L. y ForZANI, F. M. (2007) What Makes Education Research "Educational?? Educational Researcher, 36 (9), 529-540.

BÁrcena, F. (2005) La experiencia reflexiva en educación. Barcelona, Paidós.

Barrio Maestre, J. M. ${ }^{a}$ (2008) Educación y verdad. Teoría de la Educación. Revista Interuniversitaria, 20, 83-99.

BrezinKa, W. (2002) Sobre las esperanzas del educador y la imperfección de la pedagogía. Revista Española de Pedagogía, 60 (223), septiembre-diciembre, 399-414.

BRIDGES, D. (2009) Research quality assessment in education: impossible science, possible art? Bristish Educational Research Journal, 35 (4), august, 497-517.

Bridges, D.; SmeYers, P. y Smith, R. (2008) Educational Research and the Practical Judgement of Policy Makers. Journal of Philosophy of Education, 42 (1), 5-14.

BRidges, D. y Smith, R. (eds.) (2007) Philosophy, methodology and educational research. Oxford, Blackwell.

Bulterman-Bos, J. A. (2008) Will a Clinical Approach Make Education Research More Relevant for Practice? Educational Researcher, 37 (7), october, 412-420.

BurkhardT, H. y SCHOENFELd, A. H. (2003) Improving Educational Research: Toward a More Useful, More Influential, and Better-Funded Enterprise. Educational Researcher, 32 (9), december, 3-14.

CARr, W. (2004) Philosophy and Education. Journal of Philosophy of Education, 38 (1), 55-73.

- (2005) The role of theory in the professional development of an educational theorist. Pedagogy, Culture \& Society, 13 (3), 333-346.

- (2006) Education without Theory. British Journal of Educational Studies, 54 (2), 136-159. 
- (2007) Educational research as a practical science. International Journal of Research $\&$ Method in Education, 30 (3), November, 271-286.

Clemente, M. (2007) La complejidad de las relaciones teoría-práctica en educación. Teoría de la Educación. Revista Interuniversitaria, 18, 25-46.

Colom, A. J. (2007) La teoría de la educación en su doble dimensionalidad: de la realidad y del saber educativo, en Boavida, J. y García del Dujo, Á. (coords.) Teoría da educação. Contributos Ibéricos. Coimbra, Imprensa da Universidade de Coimbra, 45-70.

Colom, A. J. y Rincón, J. C. (2004) Epistemología neoidealista y fracaso fundacional del saber educativo. Teoría de la Educación. Revista Interuniversitaria, 16, 19-47.

- (2007) Narrativitat, ciéncia i educació. Barcelona, Institut D’Estudis Catalans.

Dearden, R. F. (1984) Theory and practice in education. London, Routledge and Kegal Paul.

Dewey, J. (1952) La busca de la certeza: un estudio de la relación entre el conocimiento y la acción. México, FCE.

Edmonds, E. y CAndy, L. (2010) Relating Theory, Practice and Evaluation in Practitioner Research. Leonardo, 43 (5), 470-476.

Elster, J. (1996) Tuercas y tornillos. Una introducción a los conceptos básicos de las ciencias sociales. Barcelona, Gedisa.

- (2000) El cambio tecnológico. Investigaciones sobre la racionalidad y la transformación social. Barcelona, Gedisa.

Escámez, J. (2007) Las aportaciones de la teoría de la educación. Revista Española de Pedagogía, 65 (237), 217-236.

Gadamer, H.-G. (1977) Verdad y método. Fundamentos de una hermenéutica filosófica. Salamanca, Sígueme.

García Aretio, L.; Ruiz Corbella, M. y García Blanco, M. (2009) Claves para la educación: actores, agentes y escenarios en la sociedad actual. Madrid, Narcea.

García Carrasco, J. y García del Dujo, A. (2001) La teoría de la educación en la encrucijada. Teoría de la Educación. Revista Interuniversitaria, 13, 15-43.

García del Dujo, Á.; García Carrasco, J. y Asensio, J. M. (2007) Hechos y pensamientos sobre educación en busca de una teoría: la trama entre biología, tecnología y cultura, en Boavida, J. y García del Dujo, Á. (coords.) Teoría da educação. Contributos Ibéricos. Coimbra, Imprensa da Universidade de Coimbra, 203-216.

GEERTZ, Cl. (1992) La interpretación de las culturas. Barcelona, Gedisa.

GIL, F. (2001) Educación y crisis del sujeto. Teoría de la Educación. Revista Interuniversitaria, 13, 45-68.

- (2003a) Elaboración de una teoría pedagógica de los derechos humanos, en Ruzz CorBELLA, M. (coord.) Educación moral: aprender a ser, aprender a convivir. Barcelona, Ariel, 71-89.

- (2003b) La reconstrucción de la Teoría de la Educación, Adenda XXII Seminario Interuniversitario de Teoría de la Educación, "Otros lenguajes en educación". Sitges, Universidad de Barcelona, noviembre. http://www.ucm.es/info/site/site22.html.

- (2008) Ciudadanía y humanidad. La educación en el disenso. Teoría de la Educación. Revista Interuniversitaria, 20, 25-44.

Gil, F. y Jover, G. (2007) Memoria y prospectiva de la teoría de la educación, en BoAvidA, J. y García del Dujo, Á. (coords.) Teoría da educação. Contributos Ibéricos. Coimbra, Imprensa da Universidade de Coimbra, 217-244. 
«EDUCACIÓN CON TEORÍA”. REVISIÓN PEDAGÓGICA DE LAS RELACIONES...

GonzÁlez Faraco, J. C. y Gramigna, A. (2009) Narraciones y metáforas en la construcción del pensamiento científico y la epistemología educativa. Teoría de la Educación. Revista Interuniversitaria, 22 (2), 79-94.

GonzÁlez Pérez, V. (2010) Hacia una reconstrucción de la razón pedagógica. Teoría de la Educación. Revista Interuniversitaria, 22 (2), 19-42.

Hammersley, M. (2008) Troubling criteria: a critical commentary on Furlong and Oancea's framework for assessing educational research. British Educational Research Journal, 34 (6), 747-762.

Hinst, P. H. (1963) Philosophy and Educational Theory. British Journal of Educational Studies, 12 (1), 51-64.

- (1983) Educational theory, en HisRst, P. H. (ed.) Educational theory and its foundation disciplines. London, Routledge and Kegan Paul, 3-29.

Ibáñez-Martín, J. A. (2007) Convicciones pedagógicas y desarrollo de la personalidad de mujeres y varones. Revista Española de Pedagogía, 65 (238), septiembre-diciembre, 479-516.

Jorgensen, E. R. (2005) Four Philosophical Models of the Relation Between Theory and Practice. Philosophy of Music Education Review, 13 (1), 21-36.

Jover, G. (2003) Elementos para una narrativa de la teoría de la educación, Adenda XXII Seminario Interuniversitario de Teoría de la Educación, "Otros lenguajes en educación". Sitges, Universidad de Barcelona, noviembre. http://www.ucm.es/info/site/site22. html.

- (2006) La construcció disciplinària del coneixement teóric de l'educació: crónica de discontinuitats en tres actes i epíleg. Temps d'Educació, 31, 85-102.

Jover, G. y Thoilliez, B. (2010) Cuatro décadas de Teoría de la Educación. ¿Una ecuación imposible? Teoría de la Educación. Revista Interuniversitaria, 22 (1), 43-64.

Kemis, S. (2009) Researching educational praxis: spectator and participant perspectives, en Research in Educational Praxis Symposium, Faculty of Education, Utrecht University of Applied Sciences, The Netherlands, october 7, 2009. www.ipd.gu.se/digitalassets/1286 /1286326_091028kemmis.pdf (Última consulta: 24-XI-2010).

Labaree, D. F. (2003) The Peculiar Problems of Preparing Educational Researchers. Educational Researcher, 32 (4), may, 13-22.

- (2008) The Dysfunctional Pursuit of Relevance in Education Research. Educational Researcher, 37 (7), october, 421-423.

Lagemann, E. C. (2008) Education Research as a Distributed Activity across Universities. Educational Researcher, 37 (7), 424-428.

LaGo, I. (2008) La lógica de la explicación en las ciencias sociales. Una introducción metodológica. Madrid, Alianza Editorial.

Lanceros, P. (2006) La modernidad cansada. Y otras fatigas. Madrid, Biblioteca Nueva.

Martínez, M. (2005) Estudios y profesiones educativas, en Ruiz Berrio, J. (ed.) Pedagogía y educación ante el siglo XXI. Madrid, Departamento de Teoría e Historia de la Educación, 669-678.

Moore, T. W. (1980) Introducción a la Teoría de la Educación. Madrid, Alianza.

OAnceA, A. (2005) Criticisms of educational research: key topics and levels of analysis. British Educational Research Journal, 31 (2), 157-183.

- (2007) From Procrustes to Proteus: trends and practices in the assessment of education research. International Journal of Research and Method in Education, 30 (3), 243-269. 
OAncea, A. y Furlong, J. (2007) Expressions of excellence and the assessment of applied and practice-based research. Research Papers in Education, 22 (2), 119-137.

OAncea, A. y Pring, R. (2008) The Importance of Being Thorough: On Systematic Accumulations of "What Works" in Education Research. Journal of Philosophy of Education, 42 (1), 15-39.

O'Connor, D. J. (1967) Introduction to the Philosophy of Education. London, Routledge and Kegal Paul.

QuesadA, D. (2009) Un panorama de la epistemología actual, en QuesadA, D. (coord.) Cuestiones de teoría del conocimiento. Madrid, Tecnos, 17-32.

Rodríguez NeIRA, T. (2002) Estructura y límites gnoseológicos de la "investigación-acción crítica". Revista Española de Pedagogía, 60 (223), septiembre-diciembre, 415-438.

Romero, Cl. (2006) Conocimiento, acción y racionalidad en educación. Madrid, Biblioteca Nueva.

Ruitenberg, Cl. (2009) Introduction: The Question of Method in Philosophy of Education. Journal of Philosophy of Education, 43 (3), 315-323.

SÁEz, R. (2007) La Teoría de la educación: Una búsqueda sin término en la construcción del conocimiento de la Educación. Encounters on Education, 8, fall, 109-126.

SARRAMONA, J. (2007) El futuro de la teoría de la educación en perspectiva tecnológica, en Boavida, J. y García del Dujo, Á. (coords.) Teoría da educação. Contributos Ibéricos. Coimbra, Imprensa da Universidade de Coimbra, 593-609.

Schatzki, Th. R. (2001) Introduction: practice theory, en Schatzki, Th. R.; Knorr-Cetina, K. y SAVIgny, E. von (eds.) The practice turn in contemporary theory. London, Routledge, $1-14$.

Schön, D. A. (1983) The Reflective Practitioner: How Professionals Think in Action. New York, Basic Books.

Schwab, J. J. (1969) The practical: A language for curriculum. School Review, 78, 1-24.

Sing, B. (2005) Modern Educational Theory and Practice. New Delphi, Anmol.

Smeyers, P. (2010) Repensar la filosofía de la educación. Teoría de la Educación. Revista Interuniversitaria, 22 (1), 91-116.

Smith, R. (2008) Proteus Rising: Re-Imagining Educational Research. Journal of Philosophy of Education, 42 (1), 184-198.

Sosa, E. (2009) Causalidad y epistemología, en QuesadA, D. (coord.) Cuestiones de teoría del conocimiento. Madrid, Tecnos, 111-123.

TOURIÑán, J. M. ${ }^{a}$ (1987a) Teoría de la educación. La educación como objeto de conocimiento. Madrid, Anaya.

- (1987b) Estatuto del profesorado. Función pedagógica y alternativas de formación. Madrid, Escuela Española.

- (2008) Teoría de la educación: investigación disciplinar y retos epistemológicos. Revista Internacional de Investigación Educativa, 1, 175-194.

TouriÑán, J. M. ${ }^{a}$ y SÁEz, R. (2006) La metodología de investigación y la construcción del conocimiento de la educación. Revista Galega do Ensino, 14 (48), 89-130.

Trilla, J. (2005) Hacer Pedagogía hoy, en Ruiz BerRio, J. (ed.) Pedagogía y educación ante el siglo XXI. Madrid, Departamento de Teoría e Historia de la Educación, 287-309.

Uljens, M. (2001) On General Education as a Discipline. Studies in Philosophy and Education, 20, 291-301. 
"EDUCACIÓN CON TEORÍA”. REVISIÓN PEDAGÓGICA DE LAS RELACIONES...

Van Manen, M. (1998) El tacto en la enseñanza. El significado de la sensibilidad pedagógica. Barcelona, Paidós.

Vázquez, G. (2007) La pedagogía en y de la sociedad cognitiva, en BoAvida, J. y GarCía deL Dujo, Á. (coords.) Teoría da educação. Contributos Ibéricos. Coimbra, Imprensa da Universidade de Coimbra, 259-294.

Wall, E. (2001) Educational Theory. New York, Prometheus.

Wiliam, D. (2008) What Should Education Research Do, and How Should It Do It? Educational Researcher, 37 (7), 432-438.

Winkle-Wagner, R.; Hunter, Ch. A. y Hinderliter, D. (2009) Bridging the Gap Between Theory and Practice in Educational Research. Methods at the Margins. London, MacMillan. 